\title{
Homogenization of a class of integro-differential equations with Lévy operators.
}

\author{
Mariko Arisawa \\ Wolfgang Pauli Institute, University of Wien \\ Nordbergstrasse 15, A1090 Wien, Austria \\ E-mail: mariko.arisawa@univie.ac.at
}

\section{Introduction.}

We study the periodic homogenization of

$$
\begin{gathered}
u_{\varepsilon}-c\left(\frac{x}{\varepsilon}\right) \int_{z \in \mathbf{R}^{\mathbf{N}}}\left[u_{\varepsilon}(x+z)-u_{\varepsilon}(x)-\mathbf{1}_{|z| \leq 1}\left\langle\nabla u_{\varepsilon}(x), z\right\rangle\right] q(z) d z-g\left(\frac{x}{\varepsilon}\right)=0 \quad x \in \Omega, \\
u_{\varepsilon}(x)=\phi(x) \quad x \in \Omega^{c}
\end{gathered}
$$

where the integral term, the Lévy operator, has the symmetric density

$$
q(z)=\frac{1}{|z|^{N+\alpha}} \quad z \in \mathbf{R}^{\mathbf{N}}, \quad \alpha \in(0,2) \quad \text { a constant }
$$

$\Omega$ a bounded domain in $\mathbf{R}^{\mathbf{N}}, c(\cdot)$ and $g(\cdot)$ real valued, periodic, continuous functions in $\mathbf{T}^{\mathbf{N}}, c(x)>\exists c_{0}>0$, and $\phi$ a continuous function defined in $\Omega^{c}$. We consider (11)-(2) in the framework of viscosity solutions for the integrodifferential equation (PIDE in short), introduced and studied in A. Sayah [17], O. Alvarez and A. Tourin [1], G. Barles, R. Buckdahn and E. Pardoux [7], H. Pham [16], M. Arisawa [2], 3], 44, 5], E. Jacobsen and K. Karlsen [14] and G. Barles and C. Imbert [8]. See M. Crandall, H. Ishii and P.-L. Lions [11], too. The comparaison and the existence of solutions have been 
proved in the above works. Recently in [5], the equivalence of several existing definitions was proved (see Definitions 1.1 and 1.2 in below). The homogenization means to get the unique $\operatorname{limit}_{\lim _{\varepsilon \rightarrow 0}} u_{\varepsilon}=\bar{u}$, and to characterize $\bar{u}$ by its effective equation. In mathematical finances, (1) and its evolutionary form are used in the stochastic volatility model with jump processes (see for example R. Cont and P. Tankov [10], J.P. Fouque, G. Papanicolaou, and K. Sircar [13.) We use the formal asymptotic expansion method introduced by A. Bensoussan, J. L. Lions and G. Papanicolaou 9] for linear PDEs, and then extended to nonlinear problems by P.-L. Lions, G. Papanicolaou and S. Varadhan [15] (for first-order PDEs ), L.C. Evans [12] (for second-order PDEs), in the framework of viscosity solutions. We shall derive the effective PIDE for $\bar{u}$, rigorously. First, we remind two equivalent definitions of viscosity solutions for a class of PIDEs including (1):

$$
A\left(x, u(x), \nabla u(x), \nabla^{2} u(x), I[u](x)\right)=0 \quad x \in \Omega,
$$

where $A(x, u, p, Q, I) \in C\left(\Omega \times \mathbf{R} \times \mathbf{R}^{\mathbf{N}} \times \mathbf{S}^{\mathbf{N}} \times \mathbf{R}\right), I[u](x)=\int_{z \in \mathbf{R}^{\mathbf{N}}}[u(x+z)-$ $\left.u(x)-\mathbf{1}_{|z| \leq 1}\langle\nabla u(x), z\rangle\right] q(z) d z$. For an upper (resp. lower) semicontinuous function $u \in U S C\left(\mathbf{R}^{\mathbf{N}}\right)$ (resp. $\left.L S C\left(\mathbf{R}^{\mathbf{N}}\right)\right),(p, X) \in \mathbf{R}^{\mathbf{N}} \times \mathbf{S}^{\mathbf{N}}$ is a sub(resp. super)-differential of $u$ at $x$ : if for any $\delta>0$ there exists $\varepsilon>0$ such that

$$
u(x+z)-u(x) \leq(\text { resp. } \geq)\langle p, z\rangle+\frac{1}{2}\langle X z, z\rangle+(\text { resp. }-) \delta|z|^{2} \quad \forall|z| \leq \varepsilon,
$$

Denote the set of all subdifferentials (resp. superdifferentials) of $u$ at $x$ $J_{\mathbf{R}^{\mathbf{N}}}^{2,+} u(x)\left(\operatorname{resp} . J_{\mathbf{R}^{\mathbf{N}}}^{2,-} u(x)\right)$. Set $I_{\nu, \delta}^{1,+}[u, p, X](x)=\int_{|z| \leq \nu} \frac{1}{2}\langle(X+2 \delta I) z, z\rangle q(z) d z$ (resp. $\left.I_{\nu, \delta}^{1,-}[u, p, X](x)=\int_{|z| \leq \nu} \frac{1}{2}\langle(X-2 \delta I) z, z\rangle q(z) \bar{d} z\right)$, and

$$
I_{\nu, \delta}^{2}[u, p, X](x)=\int_{|z|>\nu}\left[u(x+z)-u(x)-\mathbf{1}_{|z| \leq 1}\langle p, z\rangle\right] q(z) d z .
$$

We use the following two equivalent definitions (see [5]).

Definition 1.1. A function $u \in U S C\left(\mathbf{R}^{\mathbf{N}}\right)$ (resp. $L S C\left(\mathbf{R}^{\mathbf{N}}\right)$ ) is a viscosity subsolution (resp. supersolution) of (4), if for any $\hat{x} \in \Omega$, any $(p, X) \in J_{\mathbf{R}^{\mathbf{N}}}^{2,+} u(\hat{x})$ (resp. $\left.J_{\mathbf{R}^{\mathbf{N}}}^{2,-} u(\hat{x})\right)$, and any pair of numbers $(\varepsilon, \delta)$ satisfying (5), the following holds

$$
A\left(\hat{x}, u(\hat{x}), p, X, I_{\nu, \delta}^{1,+}\left(\operatorname{resp} . I_{\nu, \delta}^{1,-}\right)[u, p, X](\hat{x})+I_{\nu, \delta}^{2}[u, p, X](\hat{x})\right) \leq(\text { resp. } \geq) 0 .
$$

If $u$ is a subsolution and a supersolution, it is called a viscosity solution. 
Definition 1.2. A function $u \in U S C\left(\mathbf{R}^{\mathbf{N}}\right)$ (resp. $L S C\left(\mathbf{R}^{\mathbf{N}}\right)$ ) is a viscosity subsolution (resp. supersolution) of (4), if for any $\hat{x} \in \Omega$, any $\phi \in C^{2}\left(\mathbf{R}^{\mathbf{N}}\right)$ such that $u(\hat{x})=\phi(\hat{x})$ and $u-\phi$ takes a global maximum (resp. minimum) at $\hat{x}$,

$$
A\left(\hat{x}, u(\hat{x}), \nabla \phi(\hat{x}), \nabla^{2} \phi(\hat{x}), I[\phi](\hat{x})\right) \leq(\text { resp. } \geq) 0 .
$$

If $u$ is a subsolution and a supersolution, it is called a viscosity solution.

We sometimes abbreviate "viscosity" to note a (sub or super) solution. The problem (11) was chosen for simplicity to illustrate the method. The various generalizations are possible, namely to the nonlinear problem:

$$
u_{\varepsilon}+H\left(\frac{x}{\varepsilon}, \nabla u_{\varepsilon}, \nabla^{2} u_{\varepsilon}, I\left[u_{\varepsilon}\right](x)\right)=0 .
$$

\section{Formal asymptotic expansions.}

Let $u_{\varepsilon}$ be the solution of (1), and assume that

$$
u_{\varepsilon}(x)=\bar{u}(x)+\varepsilon^{\alpha} v\left(\frac{x}{\varepsilon}\right)+o\left(\varepsilon^{\alpha}\right) \quad \forall x \in \mathbf{R}^{\mathbf{N}} .
$$

Formally, $\nabla u_{\varepsilon}(x)=\nabla \bar{u}(x)+\varepsilon^{\alpha-1} \nabla_{y} v\left(\frac{x}{\varepsilon}\right), \nabla^{2} u_{\varepsilon}(x)=\nabla^{2} \bar{u}(x)+\varepsilon^{\alpha-2} \nabla_{y}^{2} v\left(\frac{x}{\varepsilon}\right)$, and by introducing them into (1), we get

$$
\begin{aligned}
& \bar{u}-c\left(\frac{x}{\varepsilon}\right) \int_{\mathbf{R}^{\mathrm{N}}}\left[\bar{u}(x+z)-\bar{u}(x)-\mathbf{1}_{|z| \leq 1}\langle\nabla \bar{u}(x), z\rangle\right] q(z) d z \\
& -c\left(\frac{x}{\varepsilon}\right) \int_{\mathbf{R}^{\mathbf{N}}} \varepsilon^{\alpha}\left[v\left(\frac{x+z}{\varepsilon}\right)-v\left(\frac{x}{\varepsilon}\right)-\mathbf{1}_{|z| \leq 1}\left\langle\nabla_{y} v\left(\frac{x}{\varepsilon}\right), \frac{z}{\varepsilon}\right\rangle\right] q(z) d z=g\left(\frac{x}{\varepsilon}\right)+o(1) .
\end{aligned}
$$

Put $y=\frac{x}{\varepsilon}$, and change the variable to $z^{\prime}=\frac{z}{\varepsilon}$. From (3)), we have

$$
\bar{u}-c(y) I[\bar{u}](x)-c(y) I[v](y)-g(y)=0 .
$$

Then, for each fixed $(x, I) \in \Omega \times \mathbf{R}(I=I[\bar{u}](x)$ in (17)), find a unique number $d(x, I)$ such that there exists a periodic solution $v(y)$ of

$$
d(x, I)-c(y) \int_{\mathbf{R}^{\mathrm{N}}}\left[v(y+z)-v(y)-\mathbf{1}_{|z| \leq 1}\left\langle\nabla_{y} v(y), z\right\rangle\right] q d z-g-c I=0,
$$

in $\mathbf{T}^{\mathbf{N}}$. In fact, the existence of $d(x, I)$ (in a weaker sense) was shown in [4] (see Theorem 3.1 in below). The effective nonlocal operator is defined as $\bar{I}(x, I)=-d(x, I)((x, I) \in \Omega \times \mathbf{R})$, and from (7), (8)), we get:

$$
\bar{u}+\bar{I}(x, I[\bar{u}](x))=0 \quad x \in \Omega,
$$

the effective equation for $\bar{u}$. Later, we justify (9) by a rigorous argument. 


\section{The derivation of the effective equation.}

To see the existence of $d(x, I)$ in (8) , consider the following

$$
l u_{l}+H\left(y, \nabla u_{l}\right)-\int_{\mathbf{R}^{\mathbf{N}}}\left[u_{l}(y+z)-u_{l}(y)-\mathbf{1}_{|z| \leq 1}\left\langle\nabla u_{l}(y), z\right\rangle\right] q d z-g=0,
$$

for $y \in \mathbf{T}^{\mathbf{N}}, l \in(0,1), H, g$ real valued functions defined in $\mathbf{T}^{\mathbf{N}} \times \mathbf{R}^{\mathbf{N}}, \mathbf{T}^{\mathbf{N}}$, periodic and Lipschitz continuous in $y$.

Theorem 3.1.([4]) Let $H(y, p)=a(y)|p|$ or 0 , where $a(\cdot) \geq \exists a_{0}>0$ periodic in $\mathbf{T}^{\mathbf{N}}$, and consider (10). The following unique number $d_{g}$ exists:

$$
\lim _{l \downarrow 0} l u_{l}(y)=d_{g} \quad y \in \mathbf{T}^{\mathbf{N}}
$$

and for any $\rho>0$, there are periodic sub and super solutions $\underline{u}$ and $\bar{u}$ of

$d_{g}+H(\nabla \underline{u}(y))+I[\underline{u}](y)-g \leq \rho, \quad d_{g}+H(\nabla \bar{u}(y))+I[\bar{u}](y)-g \geq-\rho \quad y \in \mathbf{T}^{\mathbf{N}}$.

In particular, if $N=1$ the convergence (11) is uniform, and for $\rho=0$ there exists $u=\underline{u}=\bar{u}$ which satisfies the above at the same time.

We refer the readers to [4] (Theorem 6.1) for the proof of the above result. Remark 3.1. The convergence (11) is the ergodic property (see M. Arisawa and P.-L. Lions [6] for the case of PDE). For the case of PIDE, (11) holds in more generality, e.g. for $H=H\left(x, \nabla u, \nabla^{2} u\right)$ second-order uniformly elliptic fully nonlinear operator(see [4] ). In such a case, the nonlocal homogenization (6) can be solved by the same method in this paper.

From Theorem 3.1, for any $(x, I) \in \Omega \times \mathbf{R}$, there is $\exists ! d(x, I) \in \mathbf{R}$ such that for any $\rho>0$ there exist $\underline{v}, \bar{v}$, periodic sub and super solutions of

$$
\begin{gathered}
d(x, I)+c(y) I[\underline{v}](y)-g(y)-c(y) I \leq \rho \quad y \in \mathbf{T}^{\mathbf{N}}, \\
d(x, I)+c(y) I[\bar{v}](y)-g(y)-c(y) I \geq-\rho \quad y \in \mathbf{T}^{\mathbf{N}} .
\end{gathered}
$$

Define $\bar{I}(x, I)=-d(x, I)((x, I) \in \Omega \times \mathbf{R})$. We remark the following qualitative property, the degenerate version of which was first stated in [8].

(Uniform subellipticity) There exists $\theta>0$ such that

$$
\bar{I}\left(x, I+I^{\prime}\right) \leq \bar{I}(x, I)-\theta I^{\prime} \quad \forall I^{\prime}>0, \quad \forall(x, I) \in \Omega \times \mathbf{R} .
$$


Theorem 3.2. The effective integro-differential operator $\bar{I}(x, I)$ is continuous in $\Omega \times \mathbf{R}$, and is uniformly subelliptic (12) with $\theta=c_{0}$.

Proof. The proofs are similar to the PDE's case in [12]. We do not rewrite the proof of the continuity, and mimic that of (12) for the reader's convenience. For $I^{\prime}>0, I \in \mathbf{R}, \rho>0$, from Theorem 3.1 we can take $v^{I}$, $v^{I+I^{\prime}}$ respectively a sub and a super solution of

$$
\begin{gathered}
d(x, I)-c(y) I\left[v^{I}\right](y)-g(y)-c(y) I \leq \rho \quad y \in \mathbf{T}^{\mathbf{N}} . \\
d\left(x, I+I^{\prime}\right)-c(y) I\left[v^{I+I^{\prime}}\right](y)-g(y)-c(y)\left(I+I^{\prime}\right) \geq-\rho \quad y \in \mathbf{T}^{\mathbf{N}} .
\end{gathered}
$$

By adding a constant if necessary, we may asume that $v^{I+I^{\prime}}<v^{I}$. Our goal is to prove $\bar{I}\left(x, I+I^{\prime}\right) \leq \bar{I}(x, I)-c_{0} I^{\prime}, \forall(x, I) \in \Omega \times \mathbf{R}$. Assume the contrary, i.e. there exists a constant $l>0$ such that $\bar{I}\left(x, I+I^{\prime}\right) \geq \bar{I}(x, I)-c_{0} I^{\prime}+l$, and we shall look for a contradiction. We claim that $v^{I+I^{\prime}}$ is a viscosity supersolution of

$$
-\bar{I}(x, I)-c(y) I\left[v^{I+I^{\prime}}\right](y)-g(y)-c(y) I \geq l-\rho \quad y \in \mathbf{T}^{\mathbf{N}} .
$$

To see this, assume that there exists $\phi \in C^{2}\left(\mathbf{R}^{\mathbf{N}}\right)$ such that $v^{I+I^{\prime}}-\phi$ takes a global maximum at a point $y_{0} \in \Omega, v^{I+I^{\prime}}\left(y_{0}\right)=\phi\left(y_{0}\right)$, and

$$
\phi\left(y_{0}+z\right)-\phi\left(y_{0}\right) \geq\left\langle\nabla \phi\left(y_{0}\right), z\right\rangle+\frac{1}{2}\left\langle\left(\nabla^{2} \phi\left(y_{0}\right)-2 \delta I\right) z, z\right\rangle \quad \forall|z| \leq \nu .
$$

Since $v^{I+I^{\prime}}$ is the supersolution of (14), by Definition 1.1,

$$
\begin{gathered}
-\bar{I}\left(x, I+I^{\prime}\right)-c\left(y_{0}\right) \int_{|z| \leq \nu} \frac{1}{2}\left\langle\left(\nabla^{2} \phi\left(y_{0}\right)-2 \delta I\right) z, z\right\rangle q(z) d z-c\left(y_{0}\right) \int_{|z|>\nu}\left[v^{I+I^{\prime}}\left(y_{0}+z\right)\right. \\
\left.-v^{I+I^{\prime}}\left(y_{0}\right)-\mathbf{1}_{|z| \leq 1}\left\langle\nabla \phi\left(y_{0}\right), z\right\rangle\right] q(z) d z-g\left(y_{0}\right)-c\left(y_{0}\right)\left(I+I^{\prime}\right) \geq-\rho .
\end{gathered}
$$

Then, since $c\left(y_{0}\right)>c_{0}$

$$
\begin{aligned}
& -c\left(y_{0}\right) \int_{|z| \leq \nu} \frac{1}{2}\left\langle\left(\nabla^{2} \phi\left(y_{0}\right)-2 \delta I\right) z, z\right\rangle q(z) d z \\
& -c\left(y_{0}\right) \int_{|z|>\nu}\left[v^{I+I^{\prime}}\left(y_{0}+z\right)-v^{I+I^{\prime}}\left(y_{0}\right)-\mathbf{1}_{|z| \leq 1}\left\langle\nabla \phi\left(y_{0}\right), z\right\rangle\right] q(z) d z-g\left(y_{0}\right)-c\left(y_{0}\right) I
\end{aligned}
$$




$$
\begin{aligned}
& \geq-c\left(y_{0}\right) \int_{|z| \leq \nu} \frac{1}{2}\left\langle\left(\nabla^{2} \phi\left(y_{0}\right)-2 \delta I\right) z, z\right\rangle q(z) d z-c\left(y_{0}\right) \int_{|z|>\nu}\left[v^{I+I^{\prime}}\left(y_{0}+z\right)-v^{I+I^{\prime}}\left(y_{0}\right)\right. \\
& \left.-\mathbf{1}_{|z| \leq 1}\left\langle\nabla \phi\left(y_{0}\right), z\right\rangle\right] q(z) d z-g\left(y_{0}\right)-c\left(y_{0}\right)\left(I+I^{\prime}\right)+c_{0} I^{\prime} \geq \bar{I}\left(x, I+I^{\prime}\right)+c_{0} I^{\prime}-\rho \\
& \geq \bar{I}(x, I)-c_{0} I^{\prime}+c_{0} I^{\prime}+l-\rho=\bar{I}(x, I)+l-\rho,
\end{aligned}
$$

and (15) is confirmed. For $l>0$ small enough, from (15) we have

$$
l v^{I+I^{\prime}}(y)-c(y) I\left[v^{I+I^{\prime}}\right](y)-g(y)-c(y) I \geq \bar{I}(x, I)+l-2 \rho \quad \forall y \in \mathbf{T}^{\mathbf{N}},
$$

while for $l>0$ small enough, $l v^{I}-c(y) I\left[v^{I}\right](y)-g(y)-c(y) I \leq \bar{I}(x, I)+2 \rho$, in $\mathbf{T}^{\mathbf{N}}$. From the comparison ([2], [3]), by taking $\rho=\frac{l}{8}$ we get $\sup _{y \in \mathbf{T}^{\mathbf{N}}} l\left(v^{I}(y)-\right.$ $\left.v^{I+I^{\prime}}(y)\right) \leq 4 \rho-l \leq-\frac{l}{2}$, which contradicts to $v^{I+I^{\prime}}<v^{I}$. Thus, $\bar{I}$ is uniformly subelliptic.

Now, we get the effective equation for $\bar{u}=\lim _{\varepsilon \rightarrow 0} u_{\varepsilon}$ :

$$
u+\bar{I}(x, I[u](x))=0 \quad x \in \Omega
$$

with (2). The following comparison result holds.

Theorem 3.3. Let $u \in U S C\left(\mathbf{R}^{\mathbf{N}}\right)$ and $v \in L S C\left(\mathbf{R}^{\mathbf{N}}\right)$ be respectively a sub and a super solution of (16)- (20). Then, $u \leq v$ in $\Omega$.

Proof. Since $I$ is uniformly subelliptic (Theorem 3.2), the proof is quite similar to those in [2], 3] and [8] (see [4], too). So, we abbreviate it.

\section{The justification of the effective equation.}

The main result of this paper is the following.

Theorem 4.1. Let $u_{\varepsilon}$ be the solution of (11). Then, there exists a unique $\lim _{\varepsilon \rightarrow 0} u_{\varepsilon}(x)=\exists \bar{u}(x)$, which is the solution of (16)-(2).

Proof. Put $u^{*}(x)=\lim \sup _{\varepsilon \rightarrow 0, y \rightarrow x} u_{\varepsilon}(y), u_{*}(x)=\liminf _{\varepsilon \rightarrow 0, y \rightarrow x} u_{\varepsilon}(y)$. As we shall show in below in Lemma $4.2, u^{*}, u_{*}$ are respectively a sub and a super solution of (16) - (21). Then, from the comparison (Theorem 3.3), 
$u^{*} \leq u_{*}$, and $u^{*} \leq u_{*} \leq u^{*}$ leads $\exists ! \bar{u}=\lim _{\varepsilon \rightarrow 0} u_{\varepsilon}=u^{*}=u_{*}$ which is the unique solution of (16). To complete the proof, we need the following.

Lemma 4.2. Let $u_{\varepsilon}$ be the solution of (11). Then, $u^{*}$ and $u_{*}$ are respectively a sub and a super solution of (16).

Proof of Lemma 4.2. We show that $u^{*}$ is a subsolution of (16). The proof that $u_{*}$ is a supersolution is shown in parallel, and we abbreviate it. Assume that for $\phi \in C^{2}\left(\mathbf{R}^{\mathbf{N}}\right), u^{*}-\phi$ takes a global maximum at $\hat{x} \in \Omega$ and $u^{*}(\hat{x})=\phi(\hat{x})$. As usual $([11])$, we may assume that $u^{*}-\phi$ takes the global "strict" maximum at $\hat{x}$. From Definition 1.2 our goal is to show

$$
u^{*}(\hat{x})+\bar{I}(\hat{x}, I[\phi](\hat{x})) \leq 0 .
$$

We use the argument by contradiction. Assume the contrary to (17):

$$
\phi(\hat{x})+\bar{I}(\hat{x}, I[\phi](\hat{x}))=3 \gamma>0,
$$

for $\gamma>0$. Since $\bar{I}$ is continuous, there is $U_{r}(\hat{x})=\{x|| x-\hat{x} \mid<r\}$ such that

$$
\phi(x)+\bar{I}(x, I[\phi](x)) \geq \gamma>0 \quad \forall x \in U_{r}(\hat{x}) .
$$

Put $I=I[\phi](\hat{x})$. By Theorem 3.1, a unique number $d(\hat{x}, I)$ exists, and for any $\rho>0$ there exists a periodic continuous fuction $v(y)$ satisfying

$$
d(\hat{x}, I)-c I-c I[v](y)-g(y) \leq \rho, \quad d(\hat{x}, I)-c I-c I[v](y)-g(y) \geq-\rho,
$$

in $\mathbf{T}^{\mathbf{N}}$. For $\phi_{\varepsilon}(x)=\phi(x)+\varepsilon^{\alpha} v\left(\frac{x}{\varepsilon}\right)$, (18) implies that $\phi_{\varepsilon}$ is a supersolution of

$$
\phi_{\varepsilon}-c\left(\frac{x}{\varepsilon}\right) I\left[\phi_{\varepsilon}\right](x)-g\left(\frac{x}{\varepsilon}\right) \geq \gamma \quad x \in U_{r}(\hat{x}),
$$

for $r>0$ small enough, i.e. for $\psi \in C^{2}$ such that $\phi_{\varepsilon}-\psi$ attains a global minimum at $\bar{x} \in U_{r}(\hat{x}),\left(\phi_{\varepsilon}-\psi\right)(\bar{x})=0$, and we can show (Definition 1.2)

$$
\phi_{\varepsilon}(\bar{x})-c\left(\frac{\bar{x}}{\varepsilon}\right) \int_{\mathbf{R}^{\mathbf{N}}}\left[\psi(\bar{x}+z)-\psi(\bar{x})-\mathbf{1}_{|z| \leq 1}\langle\nabla \psi(\bar{x}), z\rangle\right] q(z) d z-g\left(\frac{\bar{x}}{\varepsilon}\right) \geq \gamma .
$$

For $h(y)=\frac{1}{\varepsilon^{\alpha}}(\psi-\phi)(\varepsilon y),(v-h)(y)$ attains a global minimum at $\bar{y}=\frac{\bar{x}}{\varepsilon}$, as $\phi_{\varepsilon}-\psi$ takes the global minimum at $\bar{x}$. Since $v$ is a supersolution of (19),

$$
d(\hat{x}, I)-c(\bar{y}) I-c(\bar{y}) I[h](\bar{y})-g(\bar{y}) \geq-\rho .
$$


From the assumption (18), since $I=I[\phi](\hat{x})$

$$
\begin{aligned}
& \phi(\hat{x})-c(\bar{y}) I[\phi](\hat{x}) \\
& \quad-c(\bar{y}) \int_{\mathbf{R}^{\mathbf{N}}}\left[h(\bar{y}+z)-h(\bar{y})-\mathbf{1}_{|z| \leq 1}\langle\nabla h(\bar{y}), z\rangle\right] q(z) d z-g(\bar{y}) \geq 3 \gamma-\rho .
\end{aligned}
$$

By remarking $h\left(\frac{x}{\varepsilon}\right)=\frac{1}{\varepsilon^{\alpha}}(\psi-\phi)(x), \nabla_{y} h(y)=\varepsilon^{1-\alpha} \nabla_{x}(\psi-\phi)(\varepsilon y)$, by changing the variable $y=\frac{x}{\varepsilon}$ to $x$, from (3), for $\rho=\gamma, r$ small enough, we get

$$
\phi(\hat{x})-c(\bar{y}) \int_{\mathbf{R}^{\mathbf{N}}}\left[\psi(\bar{x}+z)-\psi(\bar{x})-\mathbf{1}_{|z| \leq 1}\langle\nabla \psi(\bar{x}), z\rangle\right] q(z) d z-g(\bar{y}) \geq 2 \gamma .
$$

The claim (21) is shown, that is $\phi_{\varepsilon}$ is the supersolution of (20). From the comparison ([2], 3], 5], 8] $),\left(u_{\varepsilon}-\phi_{\varepsilon}\right)(y) \leq \max _{U_{r}^{c}(\hat{x})}\left(u_{\varepsilon}-\phi_{\varepsilon}\right)+\gamma$ for $\forall y \in U_{r}(\hat{x})$. By letting $\varepsilon$ to $0, y$ to $\hat{x}$, we have $\left(u^{*}-\phi\right)(\hat{x}) \leq \max _{U_{r}(\hat{x})^{c}}\left(u^{*}-\phi\right)+\gamma$. Since $\gamma>0$ is arbitrary $\left(u^{*}-\phi\right)(\hat{x}) \leq \max _{U_{r}(\hat{x})^{c}}\left(u^{*}-\phi\right)$. This contradicts to the assumption that $u^{*}-\phi$ takes the global strict maximum at $\hat{x}$. Therefore, (18) is false, and (17) is proved, i.e. $u^{*}$ is the subsolution of (16). As mentioned before, the supersolution property of $u^{*}$ is proved similarly.

Since we have proved Lemma 4.2, the proof of Theorem 4.1 is completed.

\section{References}

[1] O. Alvarez, and A. Tourin, Viscosity solutions of nonlinear integrodifferential equations, Ann. Inst. H. Poincaré, Anal. Non Linéaire, 13(1996), pp. 293-317.

[2] M. Arisawa, A new definition of viscosity solution for a class of secondorder degenerate elliptic integro-differential equations, IHP Analyse nonlineaire, 23(5) (2006).

[3] M. Arisawa, Corrigendum for "A new definition of viscosity solution for a class of second-order degenerate elliptic integro-differential equations", IHP Analyse nonlineaire, 24(1) (2006).

[4] M. Arisawa, A localization of the Lévy operators arising in mathematical finances, Proceedings of the 6th RITS symposium on "Stochastic processes and applications to mathematical finance" (eds. S. Ogawa, J. Akahori, S. Watanabe), World scientifics (2007). 
[5] M. Arisawa, A remark on the definitions of viscosity solutions for the integro-differential equations with Lévy operators, J. Mathématiques Pures Appl., 89 (2008), pp. 567-574.

[6] M. Arisawa and P.-L. Lions On ergodic stochastic control. Comm. Partial Differential Equations, 23(1998), no.11-12, pp.2187-2217.

[7] G. Barles, R. Buckdahn, and E. Pardoux, Backward stochastic differential equations and integral-partial differential equations, Stochastics Stochastics Rep., 60(1-2)(1997), pp.57-83.

[8] G. Barles and C. Imbert, Second order elliptic integro-differential equations, Viscosity solutions theory revisited, to appear in IHP Analyse nonlineaire, arxiv:math/0702263v1 [math.AP], 2007.

[9] A. Bensoussan, J.L. Lions, and G. Papanicolaou, Asymptotic analysis for periodic structures. North-Holland, Amsterdam, 1978.

[10] R. Cont, and P. Tankov, Financial Modeling with jump-diffusion processes, Chapman and Hall/CRC Press, 2003.

[11] M.G. Crandall, H. Ishii, and P.-L. Lions, User's guide to viscosity solutions of second order partial differential equations. Bulletin of the AMS, vol.27, no. 1 (1992).

[12] L.C. Evans, Periodic homogeneization of certain fully nonlinear partial differential equations. Proc. Roy. Soc. Edinburgh, 120 A (1992), pp.245265.

[13] J.-P. Fouque, G. Papanicolaou and K.R. Sircar, Derivatives in financial markets with stochastic volatility, Cambridge U. Press, 2000.

[14] E. Jacobsen and K. Karlsen, A "maximum principle for semicontinuous functions" applicable to integro-partial differential equations, NoDEA Nonlinear differential equations Appl., 13(2) (2006).

[15] P.-L. Lions, G. Papanicolau, and S.R.S. Varadhan, Homogeneizations of Hamilton-Jacobi equations. preprint.

[16] H. Pham, Optimal stopping of controlled jump diffusion processes: a viscosity solution appoach, J. Math. Systems Estim. Control, 8(1) 27 (1998), pp. (electronic). 
[17] A. Sayah, Equations d'Hamilton-Jacobi du premiere ordre avec termes intégro-differentieles. I. Unicité des solutions de viscosité. II. Existence de solutions de viscosité. Comm. Partial Differential Equations, 16(6-7) (1991), pp.1057-1093. 